\title{
A Supply Chain Management Study: A Review of Theoretical Models from 2014 to 2019
}

\author{
Shu-Hsien Liao \\ Department of Management Sciences, \\ Tamkang University, Danshuei Dist, New Taipei City, Taiwan 251, R.O.C. \\ Email: Michael@mail.tku.edu.tw (Corresponding Author) \\ Retno Widowati \\ Department of Management, \\ Universitas Muhammadiyah Yogyakarta, Yogyakarta, Indonesia \\ Email:wati630421@yahoo.com
}

\begin{abstract}
Supply Chain Management (SCM) refers to suppliers, manufacturers, warehouses, distribution centers, and distributors who attempt to minimize the cost of the entire supply chain system while meeting certain customer service levels. This study examines supply chain management by reviewing the development of theoretical models of supply chain management. In regard to the development of these models, this study used "supply chain management" as the keywords/article title and searched for matching articles on the following databases from 2014 to 2019: ScienceDirect, Wiley Online Library, Sage Online Journals, Taylor \& Francis Online, Springer Link, Emerald Insight and OSCM FORUM. The various types of independent variables, dependent variables, moderators, mediators, moderated mediation and mediated moderation for the research model that were designed and tested in the articles are considered as theoretical models of supply chain management. In addition, this study found supply chain management is a cross-category study issue. Ninety-seven articles are cited from forty-eight journals with twenty-five categories. This research is the first article in terms of investigating supply chain management studies using a theoretical model review based on a qualitative review research in supply chain issues. In reviewing these articles, this study categorizes past studies of supply chain management, identifies differences and indicates possible future developments in the study of SCM.
\end{abstract}

Keywords: Supply chain; Supply chain management; Supply chain management study; Theoretical model review; Literature review

\section{INTRODUCTION}

A supply chain refers to the network structure composed of raw material suppliers, producers, distributors, retailers and final consumers involved in the production and distribution of products through the connection with upstream and downstream members (Christopher, 1998). It is also a network of enterprises and enterprise departments involved in the process of material acquisition, material processing, and delivery of finished products to users (Kache \& Seuring, 2014). A supply chain can be portrayed as a leafy tree: the production company constitutes the root, the exclusive agent is the main pole, the distributor is the branches and treetops, and the green leaf safflower is the end user. With the nodes of the pole, there is a circulation of time and the context of communication is the information management system (Khalid et al., 2015). Therefore, the relationship between companies in the supply chain is similar to a food chain in biology. It can be seen from the structural model of the supply chain that the supply chain is a network chain structure, and the relationship between the node enterprises is a demand and supply relationship (Gonzalez-Loureiro et al., 2015). A supply chain has the following main characteristics: (I) Complexity: Because the spans (hierarchies) of supply chain nodes are different, a supply chain is often composed of multiple, multitype or even multi-national enterprises, so the supply chain structure model is more complicated than the structural model of a single enterprise. (II) Dynamics: Supply chain management needs to be dynamically updated due to changes in corporate strategy and adapt to market demands, which gives supply chains obvious dynamics. (III) Responsiveness: The formation, existence and reconstruction of a supply chain are all based on certain market demands. In the operation of a supply chain, the demand of users is the flow of information. Products and services in the supply chain are the driving source of the flow and capital flow operations. (IV) Intersection: A node enterprise can be the node enterprise of one supply chain, and at the same time be the node enterprise of another supply chain. Many supply chains form a cross structure, which increases the difficulty of coordination management. Therefore, a supply chain can extend from the supplier side to the customer side, forming the process and activity of supply chain management (Kembro et al., 2014; Basnet \& Seuring, 2016; Maestrini et al., 2017; Lu et al., 2018).

Supply Chain Management (SCM) refers to suppliers, manufacturers, warehouses, distribution centers, and distributors who attempt to minimize the cost of the entire supply chain system while meeting certain customer service levels. Management methods for product manufacturing, transshipment, distribution, and sales are effectively organized together. Supply chain management includes five basic elements: planning, procurement, manufacturing, distribution, and return (Giunipero et al., 2008). Supply chain management and traditional logistics management have significant differences in inventory management methods, goods flow, 
cost, information flow, risk, planning and organizational relationships. These differences make supply chain management more advantageous than traditional logistics management (Ntabe et al., 2015). Supply chain management is implemented because it is more dynamic than traditional logistics management and can bring substantial benefits to supply chain members (Wen et al., 2018). However, in order to successfully implement supply chain management, each supply chain member must have good information sharing, and be open and honest. Information sharing is not an easy task for companies pursuing different goals. When a company cooperates with its many competitors, it is especially difficult to achieve information sharing. Therefore, successful supply chain integration requires first-hand enterprise companies to agree on the following aspects: jointly recognize the service level of the final customer, jointly determine the location of the inventory in the supply chain, and the inventory level of each inventory point, and jointly promote policies and procedures that manage the supply chain as an entity (Ding et al., 2018). The background of economic globalization has expanded the content and scope of supply chain management: Global Logistics, Outsourcing, Strategic Sourcing, and Supply Chain Collaboration (CPFR, S\&OP) have been greatly developed (Jasti \& Kodali, 2015). Supply chain management is no longer only focused on the internal operations of an enterprise, but the operation of the entire industry and value chain in the global market, along with related risk management (Sustainability) and sustainability (Sustainability) (Batista et al., 2018; Mujkic et al., 2018). Thus, supply chain management methods have been developed to provide solutions and alternatives not only for academic research but also for practical application to investigate and solve specific problems in supply chains.

Why have we done this review article on the supply chain management? This is a very important question. A literature review can be a condensed summary of the source of the data and articles (Yalcin et al., 2020). Literature review can reinterpret old data or integrate new and old interpretations, and it can also trace back the development of knowledge in the academic field, which contains major development and controversies (Acerbi \& Taisch, 2020). Depending on the situation, the literature review may examine the source of the data and provide readers with relevant or most appropriate suggestions (Asghari \& Al-e-hashem, 2020). Understanding the necessity of literature review helps to understand whether researches have done is meaningful, and to know that articles can contribute to the academic field (Al Humdan et al., 2020). On the other hand, a literature review provides a reasonable explanation for past, present and future research (Treiblmaier et al., 2020). However, a little supply chain management review which focus on the issue of theoretical model review in terms of investigating different roles on statistical variables, such as independent variables, dependent variables, moderators, mediators, moderated mediators and mediated moderators. Thus, a strong reason and motivation for this article to construct a historical background and suggestion for future works might be a contribution to the study of supply chain management.

\section{METHOD}

Regarding to statistical approach on supply chain management research, a theoretical model develops, describes and clarifies a situation related to a problem, and through the data collection or literature, the relationships between variables are examined. A good theoretical model defines the important changes in the context related to the problem and theory and explains the interactive relationship between the variables (Sharma \& Nandi, 2018; González-Teruel \& Pérez-Pulido, 2020). This study examines supply chain management by reviewing the development of theoretical models of supply chain management. In regard to the development of these models, this study used "supply chain management" as the keywords/article title and searched for matching articles on the following databases from 2014 to 2019: ScienceDirect, Wiley Online Library, Sage Online Journals, Taylor \& Francis Online, Springer Link, Emerald Insight and OSCM FORUM. The various types of independent variables, dependent variables, moderators, mediators, moderated mediation and mediated moderation for the research model that were designed and tested in the articles are considered as theoretical models of supply chain management. In reviewing these articles, this study categorizes past studies of supply chain management, identifies differences and indicates possible future developments in the study of SCM.

It is found that supply chain management is a cross-category research issue. Ninety-seven articles from forty-eight journals in twenty-five categories are used, including BUSINESS; BUSINESS, FINANCE; COMPUTER SCIENCE, ARTIFICIAL INTELLIGENCE; COMPUTER SCIENCE, INFORMATION SYSTEMS; HOSPITALITY, LEISURE, SPORT \& TOURISM; INFORMATION SYSTEMS; COMPUTER SCIENCE, INTERDISCIPLINARY APPLICATIONS; ECONOMICS; ENGINEERING, INDUSTRIAL; ENGINEERING, ELECTRICAL \& ELECTRONIC; ENGINEERING, ENVIRONMENTAL; ENGINEERING, MANUFACTURING; ENVIRONMENTAL SCIENCES; ETHICS; GREEN \& SUSTAINABLE SCIENCE \& TECHNOLOGY; FOOD SCIENCE \& TECHNOLOGY; INFORMATION SCIENCE \& LIBRARY SCIENCE; MANAGEMENT; MATHEMATICS, APPLIED; OPERATIONS RESEARCH \& MANAGEMENT SCIENCE; PLANNING \& DEVELOPMENT; SOCIOLOGY; PSYCHOLOGY, EXPERIMENTAL; PSYCHOLOGY, MULTIDISCIPLINARY; and TRANSPORTATION (2019 in Web of Science) . Thus, supply chain management issues related to management, business, information systems, operational research, management science, decision science, computer sciences, social sciences, behavioral sciences and other interdisciplinary applications are a valuable research topic for reviewing theoretical models of supply chain management.

\section{THEORETICAL MODELS WITH MODERATOR DESIGNS AND TESTS}

Moderation refers to a change in the relationship between an independent variable and a dependent variable, depending on the level of a third variable, called the moderator variable. Moderating effects are also referred to as interaction 
and conditioning effects in terms of continuous and categorical variables (Edwards, 2002). A moderator variable, commonly denoted as just $M$, is a third variable affecting the strength of the relationship between a dependent and independent variable. In correlation, a moderator is a third variable affecting the correlation of two variables. In a causal relationship, if $\mathrm{x}$ is the predictor variable and $\mathrm{y}$ is an outcome variable, then $\mathrm{z}$ is the moderator variable affecting the casual relationship of $\mathrm{x}$ and $\mathrm{y}$. Most moderator variables measure causal relationships using a regression coefficient. The moderator variable, if it found to be significant, that can cause an amplifying or weakening effect between $\mathrm{x}$ and $\mathrm{y}$. In ANOVA, the moderator variable effect is represented by the interaction effect between the dependent variable and the factor variable. On the other hand, in a regression equation, when the relationship between the dependent variable and the independent variable is linear, then the dependent variable may change when the value of the moderator variable changes. In a linear relationship, the relationship represents the interaction effect of the moderator and the independent variable. When the relationship is non-linear, a significant moderator variable value does not prove to be a true moderator effect, unless the moderator is a manipulated variable (Kenny et al., 2006).

In Table 1, moderator variables are designed and tested on theoretical models for different consumer subject backgrounds and variables such as continuous and categorical moderator variables. In terms of continuous moderator variables, regression and latent change modeling with SEM, PLS, Regressions, and ANOVA are commonly used to examine a SCM theoretical model (Kaufmann \& Gaeckler, 2015). In terms of subject background, we selected and tested the following subjects: Spanish logistics firms, the UK logistics sectors, UK manufacturing plants, Hong Kong wholesale trade companies, USA consumers, international firms, German firms, Taiwanese manufacturing firms, USA manufacturing and service industries, South Korean medical service facilities, globally distributed manufacturing plants, Egyptian manufacturing firms, South Korean manufacturers, USA OEM manufacturers, India manufacturing firms, India disaster relief respondents, international logistics managers, Iranian manufacturing industries, 17 USA industries, Indian hotels and restaurants, United Arab green firms, China RosettaNet firms, UK OEM agents, supplier agents, logistics providers, Australian retail firms, Sweden SMEs and Taiwan businesses. In terms of moderators on theoretical models, we tested the following designs: logistical capabilities, firm size, supply base complexity, slack/visibility, market complexity, trust, product complexity, environmental collaboration in supply chains, firm business type, hospital size, time/relationship quality/national culture, information sharing/logistics integration, centralization/formalization, ISO 9000, supply chain dynamism, supply chain culture of competitiveness, penalties/production costs/interdependence costs, supply chain flexibility, personal innovation, relational bonding, environmental proactivity, employees' resistance to change, environmental uncertainty, business process innovation, technology orientation, HR practices/training/management commitment, participation in standards consortia, supply network topology, legal enforceability/guanxi importance, supplier integration and top management, network capability and process innovation and uncertainty.

As we can see, supply chain integration and information sharing are popular independent variables of theoretical designs. Performance plays a main dependent variable role for the consideration of theoretical models. In addition, environmental factors, TQM, supply chain scale/type/complexity/flexibility/innovation/culture and manpower are diversity developments among the moderators in the designing and testing of theoretical models. Manufacturing, logistics, service and green firms are popular industry types from the subject sources.

Table 1 Theoretical models with moderator designs and tests

\begin{tabular}{|c|c|c|c|c|c|c|}
\hline Year & Author(s) & $\begin{array}{l}\text { Independent } \\
\text { variable }\end{array}$ & Moderator & $\begin{array}{l}\text { Dependent } \\
\text { variable }\end{array}$ & Method & Subjects \\
\hline 2014 & $\begin{array}{l}\text { Wiengarten et } \\
\text { al. }\end{array}$ & $\begin{array}{l}\text { Supply chain inte- } \\
\text { gration }\end{array}$ & $\begin{array}{l}\text { Logistical ca- } \\
\text { pabilities }\end{array}$ & Performance & Regression & Spain logistics firms \\
\hline 2014 & $\begin{array}{l}\text { Ramanathan et } \\
\text { al. }\end{array}$ & $\begin{array}{l}\text { Barcode experi- } \\
\text { ence }\end{array}$ & Firm size & $\begin{array}{l}\text { Government } \\
\text { support }\end{array}$ & ANOVA & UK logistics sectors \\
\hline 2014 & $\begin{array}{l}\text { Brandon-Jones } \\
\text { et al. }\end{array}$ & $\begin{array}{l}\text { Supply chain con- } \\
\text { nectivity/ Infor- } \\
\text { mation sharing re- } \\
\text { sources }\end{array}$ & $\begin{array}{l}\text { Supply base } \\
\text { complexity }\end{array}$ & $\begin{array}{l}\text { Enhances resili- } \\
\text { ence/ Robust- } \\
\text { ness }\end{array}$ & Regression & UK manufacturing plants \\
\hline 2014 & $\begin{array}{l}\text { Brandon-Jones } \\
\text { et al. }\end{array}$ & $\begin{array}{l}\text { Supply base com- } \\
\text { plexity }\end{array}$ & Slack; Visibility & $\begin{array}{l}\text { Disruptions and } \\
\text { performance }\end{array}$ & Regression & UK manufacturing firms \\
\hline 2015 & Wong et al. & $\begin{array}{l}\text { Supply chain infor- } \\
\text { mation integration }\end{array}$ & $\begin{array}{l}\text { Market com- } \\
\text { plexity }\end{array}$ & Performance & Chi-square test & $\begin{array}{l}\text { Hong Kong wholesale } \\
\text { trade companies }\end{array}$ \\
\hline 2015 & Bonn et al. & $\begin{array}{l}\text { Consumer percep- } \\
\text { tions of sustaina- } \\
\text { ble practices }\end{array}$ & Trust & $\begin{array}{l}\text { Behavioral in- } \\
\text { tentions }\end{array}$ & $\begin{array}{l}\text { Hierarchical } \\
\text { Multiple Regres- } \\
\text { sion }\end{array}$ & USA consumers \\
\hline 2015 & $\begin{array}{l}\text { Caniato \& } \\
\text { Größler }\end{array}$ & $\begin{array}{l}\text { New product de- } \\
\text { velopment }\end{array}$ & $\begin{array}{l}\text { Product com- } \\
\text { plexity }\end{array}$ & $\begin{array}{l}\text { Supply chain } \\
\text { management in- } \\
\text { tegration }\end{array}$ & $\begin{array}{l}\text { Multivariate lin- } \\
\text { ear regression }\end{array}$ & International firms \\
\hline 2015 & Eckstein et al. & $\begin{array}{l}\text { Supply chain } \\
\text { adaptability }\end{array}$ & $\begin{array}{l}\text { Product com- } \\
\text { plexity }\end{array}$ & $\begin{array}{l}\text { Cost/ opera- } \\
\text { tional perfor- } \\
\text { mance }\end{array}$ & $\begin{array}{l}\text { Hierarchical re- } \\
\text { gression }\end{array}$ & Germany firms \\
\hline
\end{tabular}


Shu-Hsien et al:: A Supply Chain Management Study: A Review of Theoretical Models from 2014 to 2019

Table 1 (con`t) Theoretical models with moderator designs and tests

\begin{tabular}{|c|c|c|c|c|c|c|}
\hline 2015 & Chen et al. & $\begin{array}{l}\text { Environmental } \\
\text { management strat- } \\
\text { egy }\end{array}$ & $\begin{array}{l}\text { Environmental } \\
\text { collaboration in } \\
\text { supply chains }\end{array}$ & $\begin{array}{l}\text { Environment- } \\
\text { performance re- } \\
\text { lationship }\end{array}$ & Regression & $\begin{array}{l}\text { Taiwan manufacturing } \\
\text { firms }\end{array}$ \\
\hline 2016 & $\begin{array}{l}\text { Mariadoss et } \\
\text { al. }\end{array}$ & $\begin{array}{l}\text { Sustainable pur- } \\
\text { chasing practices }\end{array}$ & $\begin{array}{l}\text { Firm business } \\
\text { type }\end{array}$ & $\begin{array}{l}\text { Sustainable sup- } \\
\text { ply practices }\end{array}$ & SEM & $\begin{array}{l}\text { USA manufacturing and } \\
\text { service industries }\end{array}$ \\
\hline 2016 & Yoon et al. & $\begin{array}{l}\text { Innovation leader- } \\
\text { ship }\end{array}$ & Hospital size & SC efficiency & SEM & $\begin{array}{l}\text { South Korea medical ser- } \\
\text { vice facilities }\end{array}$ \\
\hline 2016 & Chang et al. & Internal integration & $\begin{array}{l}\text { Time; Relation- } \\
\text { ship quality; } \\
\text { National cul- } \\
\text { ture }\end{array}$ & $\begin{array}{l}\text { Financial perfor- } \\
\text { mance }\end{array}$ & SEM & $\begin{array}{l}\text { SCIE journal sample meta } \\
\text { data }\end{array}$ \\
\hline 2016 & Liu et al. & $\begin{array}{l}\text { supply chain tech- } \\
\text { nology utilization }\end{array}$ & $\begin{array}{l}\text { Information } \\
\text { sharing; Logis- } \\
\text { tics integration }\end{array}$ & $\begin{array}{l}\text { Delivery perfor- } \\
\text { mance }\end{array}$ & SEM & USA firms \\
\hline 2016 & Flynn et al. & $\begin{array}{l}\text { Supply chain inte- } \\
\text { gration }\end{array}$ & $\begin{array}{l}\text { Centralization; } \\
\text { Formalization }\end{array}$ & Uncertainty & $\begin{array}{l}\text { Hierarchical re- } \\
\text { gression }\end{array}$ & $\begin{array}{l}339 \text { globally distributed } \\
\text { manufacturing plants }\end{array}$ \\
\hline 2016 & Mokadem & Customer priorities & ISO 9000 & $\begin{array}{l}\text { Supply chain } \\
\text { alignment }\end{array}$ & $\begin{array}{l}\text { Moderated re- } \\
\text { gression }\end{array}$ & Egypt manufacturing firms \\
\hline 2016 & Lee et al. & $\begin{array}{l}\text { Supply chain inte- } \\
\text { gration }\end{array}$ & $\begin{array}{l}\text { Supply chain } \\
\text { dynamism }\end{array}$ & $\begin{array}{l}\text { Logistics perfor- } \\
\text { mance }\end{array}$ & $\begin{array}{l}\text { Moderated hier- } \\
\text { archical regres- } \\
\text { sion }\end{array}$ & $\begin{array}{l}\text { South Korean manufactur- } \\
\text { ers }\end{array}$ \\
\hline 2016 & Kim et al. & $\begin{array}{l}\text { Supply chain infor- } \\
\text { mation sharing }\end{array}$ & $\begin{array}{l}\text { Supply chain } \\
\text { culture of com- } \\
\text { petitiveness }\end{array}$ & $\begin{array}{l}\text { Supply chain } \\
\text { performance }\end{array}$ & Regression & $\begin{array}{l}\text { South Korean manufactur- } \\
\text { ers }\end{array}$ \\
\hline 2017 & Agrawal et al. & Interdependence & $\begin{array}{l}\text { Penalties; pro- } \\
\text { duction costs; } \\
\text { Interdepend- } \\
\text { ence costs }\end{array}$ & $\begin{array}{l}\text { Quality perfor- } \\
\text { mance }\end{array}$ & Regression & USA OEM manufactures \\
\hline 2017 & $\begin{array}{l}\text { Sreedevi \& Sa- } \\
\text { ranga }\end{array}$ & $\begin{array}{l}\text { Environmental un- } \\
\text { certainty }\end{array}$ & $\begin{array}{l}\text { Supply chain } \\
\text { flexibility }\end{array}$ & $\begin{array}{l}\text { Supply chain } \\
\text { risk }\end{array}$ & SEM & India manufacturing firms \\
\hline 2017 & Kabra et al. & $\begin{array}{l}\text { Performance ex- } \\
\text { pectancy }\end{array}$ & $\begin{array}{l}\text { Personal inno- } \\
\text { vation }\end{array}$ & $\begin{array}{l}\text { Effort expec- } \\
\text { tancy }\end{array}$ & PLS/SEM & $\begin{array}{l}\text { India disaster relief re- } \\
\text { spondents }\end{array}$ \\
\hline 2017 & Li \& Huang & $\begin{array}{l}\text { Green supply } \\
\text { chain practices }\end{array}$ & $\begin{array}{l}\text { Relational } \\
\text { bonding }\end{array}$ & $\begin{array}{l}\text { Green innova- } \\
\text { tion perfor- } \\
\text { mance }\end{array}$ & ANOVA & $\begin{array}{l}\text { Taiwan manufacturing } \\
\text { firms }\end{array}$ \\
\hline 2017 & Liu et al. & $\begin{array}{l}\text { External integra- } \\
\text { tion capability }\end{array}$ & $\begin{array}{l}\text { Environmental } \\
\text { proactivity }\end{array}$ & $\begin{array}{l}\text { Supplier ap- } \\
\text { praisal capability }\end{array}$ & $\begin{array}{l}\text { Hierarchical lin- } \\
\text { ear regression }\end{array}$ & $\begin{array}{l}\text { International logistics } \\
\text { managers }\end{array}$ \\
\hline 2017 & Nejati et al. & $\begin{array}{l}\text { Green human re- } \\
\text { source manage- } \\
\text { ment }\end{array}$ & $\begin{array}{l}\text { Employees' re- } \\
\text { sistance to } \\
\text { change }\end{array}$ & $\begin{array}{l}\text { Green supply } \\
\text { chain manage- } \\
\text { ment }\end{array}$ & (PLS-SEM) & $\begin{array}{l}\text { Iran manufacturing indus- } \\
\text { tries }\end{array}$ \\
\hline 2017 & Gligor & Supply chain fit & $\begin{array}{l}\text { Environmental } \\
\text { uncertainty }\end{array}$ & $\begin{array}{l}\text { Financial perfor- } \\
\text { mance }\end{array}$ & $\begin{array}{l}\text { Hierarchical } \\
\text { moderated re- } \\
\text { gression }\end{array}$ & USA 17 industries \\
\hline 2018 & Schniederjans & $\begin{array}{l}\text { Social quality man- } \\
\text { agement }\end{array}$ & $\begin{array}{l}\text { Business pro- } \\
\text { cess innovation }\end{array}$ & $\begin{array}{l}\text { Supply chain } \\
\text { performance }\end{array}$ & $\begin{array}{l}\text { Hierarchical } \\
\text { moderated re- } \\
\text { gression }\end{array}$ & USA manufacturing firms \\
\hline 2018 & Mandal & $\begin{array}{l}\text { Big data analytics } \\
\text { management ca- } \\
\text { pabilities }\end{array}$ & $\begin{array}{l}\text { Technology ori- } \\
\text { entation }\end{array}$ & $\begin{array}{l}\text { Sustainable } \\
\text { tourism supply } \\
\text { chain perfor- } \\
\text { mance }\end{array}$ & PLS & $\begin{array}{l}\text { India hotels and restau- } \\
\text { rants }\end{array}$ \\
\hline 2018 & $\begin{array}{l}\text { El-Kassar \& } \\
\text { Singh }\end{array}$ & $\begin{array}{l}\text { Green innovation; } \\
\text { Big Data Adoption }\end{array}$ & $\begin{array}{l}\text { HR practices, } \\
\text { training/Man- } \\
\text { agement com- } \\
\text { mitment }\end{array}$ & $\begin{array}{l}\text { Organizational } \\
\text { performance }\end{array}$ & (PLS-SEM) & United Arab green firms \\
\hline 2018 & Xu et al. & Knowledge sharing & $\begin{array}{l}\text { Participation in } \\
\text { standards con- } \\
\text { sortia }\end{array}$ & $\begin{array}{l}\text { Process integra- } \\
\text { tion }\end{array}$ & PLS & China RosettaNet firms \\
\hline
\end{tabular}


Table 1 (con`t) Theoretical models with moderator designs and tests

\begin{tabular}{|l|l|l|l|l|l|l|}
\hline 2018 & Ledwoch et al. & Effectiveness & $\begin{array}{l}\text { Supply network } \\
\text { topology }\end{array}$ & $\begin{array}{l}\text { Risk manage- } \\
\text { ment strategies }\end{array}$ & $\begin{array}{l}\text { Simple expo- } \\
\text { nential smooth- } \\
\text { ing forecasting } \\
\text { method }\end{array}$ & $\begin{array}{l}\text { UK OEM agents, supplier } \\
\text { agents, logistics providers }\end{array}$ \\
\hline 2018 & Yang et al. & Exchange hazards & $\begin{array}{l}\text { Legal enforce- } \\
\text { ability; Guanxi } \\
\text { importance }\end{array}$ & Opportunism & Regression & China manufacturing firms \\
\hline 2018 & Shou et al. & $\begin{array}{l}\text { Supply chain risk } \\
\text { management }\end{array}$ & $\begin{array}{l}\text { Supplier inte- } \\
\text { gration }\end{array}$ & $\begin{array}{l}\text { Operational per- } \\
\text { formance }\end{array}$ & SEM & International survey \\
\hline 2018 & Shee et al. & $\begin{array}{l}\text { Supplier and inter- } \\
\text { nal integration }\end{array}$ & $\begin{array}{l}\text { Top manage- } \\
\text { ment }\end{array}$ & $\begin{array}{l}\text { Supply chain } \\
\text { performance }\end{array}$ & SEM & Australian retail firms \\
\hline 2019 & Partanen et al. & $\begin{array}{l}\text { Supply chain ambi- } \\
\text { dexterity }\end{array}$ & $\begin{array}{l}\text { Network capa- } \\
\text { bility }\end{array}$ & $\begin{array}{l}\text { Manufacturing } \\
\text { SME perfor- } \\
\text { mance: }\end{array}$ & SEM & Sweden SMEs \\
\hline 2019 & $\begin{array}{l}\text { Gokarn and } \\
\text { Kuthambalayan }\end{array}$ & Firms' capabilities & $\begin{array}{l}\text { Business systems } \\
\text { leveraging }\end{array}$ & $\begin{array}{l}\text { Process inno- } \\
\text { vation and un- } \\
\text { certainty }\end{array}$ & $\begin{array}{l}\text { Supply chain } \\
\text { performance }\end{array}$ & SEM \\
\hline
\end{tabular}

\section{THEORETICAL MODELS WITH MEDIATOR DESIGNS AND TEST}

Mediation seeks to identify and explain the mechanism or process underlying an observed relationship between an independent variable and a dependent variable via the inclusion of a third hypothetical variable, known as a mediator variable (also a mediating variable, intermediary variable, or intervening variable). A mediator variable is the variable that causes mediation in the dependent and independent variables. In other words, it explains the relationship between the dependent variable and independent variable. The process of complete mediation is defined as the complete intervention caused by the mediator variable. The results in the initial variable no longer affect the outcome variable. The process of partial mediation is defined as partial intervention. The mediation caused by the mediator variable is developed as a mediation model. This model, which develops due to mediation, is a causal model. In other words, this means the mediator variable has been assumed to cause an effect in the outcome variable and not vice versa. Once we find a cause-effect relationship, the next question is related to the mechanisms of change (Baron \& Kenny, 1986). On the other hand, mediators play a prominent role in establishing these mechanisms because they are variables that intervene between the cause and its effect. A wide range of designs can focus on mediators. These include both non-experimental designs (i.e., cross-sectional or longitudinal) and experimental designs. The mediation caused by the variable cannot be defined statistically. On the contrary, statistics can be utilized to assess an assumed meditational model developed by the mediator variable. Experimental designs are more appropriate designs for studying mediation but no single study can meet all prescriptive requirements for considering a specific mediator as a causal mechanism. A strong recommendation is that any endeavor to establish mediation should be complemented by a conceptual analysis through the lens of a well-supported and/or wellspecified theory (David \& Sava, 2015).
Table 2 shows structural equation modeling (SEM) and regression are the most common methods used to examine consumer behavior mediation models. Structural equation modeling is a multivariate statistical analysis technique used to analyze structural relationships. This technique combines factor analysis and multiple regression analysis and is used to analyze the structural relationship between measured variables and latent constructs. This method is preferred by this researcher because it estimates multiple and interrelated dependencies in a single analysis. In this analysis, two types of variables are used: endogenous variables and exogenous variables. Endogenous variables are equivalent to dependent variables and are equal to the independent variable. There are two types of SEM models: a measurement model and a structural model. These are classified in terms of testing the proposed casual relationships (Mueller, 1996). Several software packages can fit structural equation models. LISREL was the first such software. It was initially released in the 1970s. There are also several packages for the R open source statistical environment. The OpenMx R package provides an open source and enhanced version of the Mx application (Mplus) (Kline, 2015).

In terms of subject background, we examine Chinese manufacturers, European companies, South Korean manufacturers, Taiwanese TFT-LCD industry, Spanish manufacturers, Swiss manufacturing firms, US technology firms, US purchasing and supply chain managers, Chinese hotels, Indian manufacturing firms, European manufacturing sectors and firms, Chinese IT firms, Taiwan's networking communication industry, Australian courier firms, Global oil \& gas firms, Malaysia's manufacturing industry, French sustainability firms, Pakistani manufacturing companies, Australian manufacturing firms, Pakistan public-owned companies and USA supply chain professionals and procurement specialists etc. In terms of the mediator variable, we investigated the following designs and tests: efficiency, project-level factors, supply chain integration, supply chain capabilities, customer satisfaction/organizational performance, supply chain coordination, Guanxi, purchasing costs/supply chain sustainability 
risk costs/cooperation benefits/benefits stemming, championing behavior, resource reconfiguration, E-business integration, relational governance, supply chain quality, relationship strength, contract management, internal and external processes, supply chain capability, knowledge integration, supply chain performance, knowledge sharing, supply chain uncertainty and risk, supply chain agility, top management commitment, collaborative supply chain capabilities, supplier development and internal impetus Supply chain agility, and Organizational culture.
This study found that supply chain collaboration, trust and innovation are popular independent variables in theoretical designs. Performance plays a main dependent variable role for consideration in theoretical models. In addition, supply chain capability, integration and quality are diversity developments among the design and test moderators in theoretical models. Manufacturing, technology and service firms are popular industry types in the subject sources.

Table 2 Theoretical models with mediator designs and tests

\begin{tabular}{|c|c|c|c|c|c|c|}
\hline Year & Author(s) & $\begin{array}{l}\text { Independent varia- } \\
\text { ble }\end{array}$ & Mediator & $\begin{array}{l}\text { Dependent varia- } \\
\text { ble }\end{array}$ & Method & Subjects \\
\hline 2014 & Yang & Agility & Cost efficiency & Performance & SEM & China manufacturers \\
\hline 2014 & Brinkhoff et al. & Trust & Project-level factors & Project success & $\begin{array}{l}\text { PLS/ } \\
\text { SEM }\end{array}$ & European companies \\
\hline 2014 & Seo et al. & Innovativeness & $\begin{array}{l}\text { Supply chain integra- } \\
\text { tion }\end{array}$ & $\begin{array}{l}\text { Supply chain per- } \\
\text { formance }\end{array}$ & SEM & $\begin{array}{l}\text { South Korean manu- } \\
\text { facturers }\end{array}$ \\
\hline 2014 & Liao \& Kuo & $\begin{array}{l}\text { Supply chain collab- } \\
\text { oration }\end{array}$ & $\begin{array}{l}\text { Supply chain capabil- } \\
\text { ities }\end{array}$ & Firm performance & SEM & $\begin{array}{l}\text { Taiwan TFT-LCD in- } \\
\text { dustry }\end{array}$ \\
\hline 2015 & $\begin{array}{l}\text { Gómez- } \\
\text { Cedeño et et. }\end{array}$ & $\begin{array}{l}\text { Human resource } \\
\text { management }\end{array}$ & $\begin{array}{l}\text { Customer satisfac- } \\
\text { tion; Organizational } \\
\text { performance }\end{array}$ & SCM outcomes & SEM & Spain manufacturers \\
\hline 2015 & Huo et al. & $\begin{array}{l}\text { Relationship com- } \\
\text { mitment }\end{array}$ & $\begin{array}{l}\text { Supply chain coordi- } \\
\text { nation }\end{array}$ & $\begin{array}{l}\text { Supply chain per- } \\
\text { formance }\end{array}$ & SEM & $\begin{array}{l}\text { China manufacturing } \\
\text { firms }\end{array}$ \\
\hline 2015 & Luo et al. & $\begin{array}{l}\text { Asset specificity; } \\
\text { Volume uncertainty; } \\
\text { Environmental com- } \\
\text { petition }\end{array}$ & Guanxi & $\begin{array}{l}\text { Green supply } \\
\text { chain collaboration }\end{array}$ & PLS/SEM & $\begin{array}{l}\text { Chinese manufactur- } \\
\text { ing organizations }\end{array}$ \\
\hline 2015 & Busse & $\begin{array}{l}\text { Sustainability-re- } \\
\text { lated conditions }\end{array}$ & $\begin{array}{l}\text { Purchasing costs; } \\
\text { Supply chain sustain- } \\
\text { ability risk costs; Co- } \\
\text { operation benefits; } \\
\text { Benefits stemming }\end{array}$ & $\begin{array}{l}\text { Economic perfor- } \\
\text { mance }\end{array}$ & SEM & $\begin{array}{l}\text { Swiss manufacturing } \\
\text { firms }\end{array}$ \\
\hline 2015 & $\begin{array}{l}\text { Wichmann et } \\
\text { al. }\end{array}$ & Commitment & $\begin{array}{l}\text { Championing behav- } \\
\text { ior }\end{array}$ & Network centrality & SEM & U.S. technology firms \\
\hline 2015 & Ambulkar et al. & $\begin{array}{l}\text { Supply chain disrup- } \\
\text { tion orientation }\end{array}$ & $\begin{array}{l}\text { Resource reconfigu- } \\
\text { ration }\end{array}$ & Firm resilience & SEM & $\begin{array}{l}\text { USA manufacturing } \\
\text { firms }\end{array}$ \\
\hline 2015 & Shi \& Liao & $\begin{array}{l}\text { Inter-firm depend- } \\
\text { ence; Inter-firm trust }\end{array}$ & $\begin{array}{l}\text { E-business integra- } \\
\text { tion }\end{array}$ & $\begin{array}{l}\text { Operational perfor- } \\
\text { mance }\end{array}$ & SEM & $\begin{array}{l}\text { China manufacturing } \\
\text { firms }\end{array}$ \\
\hline 2016 & Singh \& Teng & Trust & $\begin{array}{l}\text { Relational govern- } \\
\text { ance }\end{array}$ & $\begin{array}{l}\text { Supply chain per- } \\
\text { formance }\end{array}$ & SEM & $\begin{array}{l}\text { USA purchasing and } \\
\text { supply chain manag- } \\
\text { ers }\end{array}$ \\
\hline 2016 & Zhong et al. & $\begin{array}{l}\text { Quality manage- } \\
\text { ment }\end{array}$ & Supply chain quality & Hotel performance & SEM & China hotels \\
\hline 2016 & Kumar et al. & $\begin{array}{l}\text { Collaborative cul- } \\
\text { ture }\end{array}$ & Relationship strength & $\begin{array}{l}\text { Supply chain per- } \\
\text { formance }\end{array}$ & $\begin{array}{l}\text { PLS/ } \\
\text { SEM }\end{array}$ & $\begin{array}{l}\text { India manufacturing } \\
\text { firms }\end{array}$ \\
\hline 2016 & $\begin{array}{l}\text { Bode \& Mac- } \\
\text { donald }\end{array}$ & Disruption & $\begin{array}{l}\text { Disruption recogni- } \\
\text { tion; disruption diag- } \\
\text { nosis; response de- } \\
\text { velopment; response } \\
\text { implementation }\end{array}$ & $\begin{array}{l}\text { Supply chain com- } \\
\text { plexity }\end{array}$ & $\begin{array}{l}\text { OLS re- } \\
\text { gression }\end{array}$ & $\begin{array}{l}\text { Europe manufactur- } \\
\text { ing sectors and firms }\end{array}$ \\
\hline 2016 & $\begin{array}{l}\text { Pradhan \& } \\
\text { Routroy }\end{array}$ & Risk management & $\begin{array}{l}\text { Contract manage- } \\
\text { ment }\end{array}$ & SM performance & SEM & $\begin{array}{l}\text { India manufacturing } \\
\text { firms }\end{array}$ \\
\hline 2016 & Peng et al. & $\begin{array}{l}\text { Information technol- } \\
\text { ogy }\end{array}$ & $\begin{array}{l}\text { Internal and External } \\
\text { processes }\end{array}$ & Firm performance & SEM & China IT firms \\
\hline 2017 & Liao et al. & $\begin{array}{l}\text { Supply chain collab- } \\
\text { oration value inno- } \\
\text { vation }\end{array}$ & $\begin{array}{l}\text { Supply chain capabil- } \\
\text { ity }\end{array}$ & $\begin{array}{l}\text { Competitive ad- } \\
\text { vantage }\end{array}$ & SEM & $\begin{array}{l}\text { Taiwan networking } \\
\text { communication in- } \\
\text { dustry }\end{array}$ \\
\hline
\end{tabular}


Table 2 (con`t) Theoretical models with mediator designs and tests

\begin{tabular}{|c|c|c|c|c|c|c|}
\hline 2017 & Durach et al. & Risk management & Guanxi & $\begin{array}{l}\text { Supplier relation- } \\
\text { ships }\end{array}$ & SEM & China suppliers \\
\hline 2017 & Li et al. & $\begin{array}{l}\text { Market-oriented en- } \\
\text { vironmental sustain- } \\
\text { ability }\end{array}$ & $\begin{array}{l}\text { Knowledge integra- } \\
\text { tion }\end{array}$ & Performance & SEM & Chinese exporters \\
\hline 2017 & Gandhi et al. & $\begin{array}{l}\text { Supply chain man- } \\
\text { agement practices }\end{array}$ & $\begin{array}{l}\text { Supply chain perfor- } \\
\text { mance }\end{array}$ & Firm performance & $\begin{array}{l}\text { Multiple re- } \\
\text { gression }\end{array}$ & India firms \\
\hline 2017 & Wang \& $\mathrm{Hu}$ & $\begin{array}{l}\text { Collaborative inno- } \\
\text { vation activities }\end{array}$ & Knowledge sharing & $\begin{array}{l}\text { Innovation perfor- } \\
\text { mance }\end{array}$ & $\begin{array}{l}\text { Hierar- } \\
\text { chical Mul- } \\
\text { tiple Re- } \\
\text { gression }\end{array}$ & China manufacturers \\
\hline 2017 & Chan et al. & Strategic flexibility & Supply chain agility & $\begin{array}{l}\text { Manufacturing flex- } \\
\text { ibility }\end{array}$ & SEM & $\begin{array}{l}\text { China garment man- } \\
\text { ufacturers }\end{array}$ \\
\hline 2018 & Ebrahimi et al. & $\begin{array}{l}\text { Supply chain inte- } \\
\text { gration }\end{array}$ & $\begin{array}{l}\text { Internal, customer; } \\
\text { Supplier integration }\end{array}$ & $\begin{array}{l}\text { Operational perfor- } \\
\text { mance }\end{array}$ & SEM & Global oil \& gas firms \\
\hline 2018 & Sundram et al. & $\begin{array}{l}\text { Supply chain infor- } \\
\text { mation manage- } \\
\text { ment }\end{array}$ & $\begin{array}{l}\text { Supply chain integra- } \\
\text { tion }\end{array}$ & $\begin{array}{l}\text { Manufacturing per- } \\
\text { formance }\end{array}$ & $\begin{array}{l}\text { Mediated } \\
\text { multiple re- } \\
\text { gression } \\
\end{array}$ & $\begin{array}{l}\text { Malaysia manufactur- } \\
\text { ing industry }\end{array}$ \\
\hline 2018 & Saeed et al. & Product modularity & Supply chain agility & $\begin{array}{l}\text { Responsiveness; } \\
\text { Cost reduction. }\end{array}$ & SEM & $\begin{array}{l}\text { USA manufacturing } \\
\text { firms }\end{array}$ \\
\hline 2018 & Dubey et al. & External pressures & $\begin{array}{l}\text { Top management } \\
\text { commitment }\end{array}$ & $\begin{array}{l}\text { Supplier relation- } \\
\text { ship management }\end{array}$ & $\begin{array}{l}\text { Hierar- } \\
\text { chical re- } \\
\text { gression }\end{array}$ & $\begin{array}{l}\text { France sustainability } \\
\text { firms }\end{array}$ \\
\hline 2018 & $\begin{array}{l}\text { Sadiq Jajja et } \\
\text { al. }\end{array}$ & Supply chain risk & $\begin{array}{l}\text { Supply chain integra- } \\
\text { tion }\end{array}$ & Agility performance & SEM & $\begin{array}{l}\text { Pakistan manufactur- } \\
\text { ing companies }\end{array}$ \\
\hline 2018 & $\begin{array}{l}\text { Levi-Bliech et } \\
\text { al. }\end{array}$ & Mobile technology & $\begin{array}{l}\text { Collaborative supply } \\
\text { chain capabilities }\end{array}$ & $\begin{array}{l}\text { Business process } \\
\text { performance }\end{array}$ & SEM & $\begin{array}{l}\text { Six European country } \\
\text { managers }\end{array}$ \\
\hline 2018 & Wang et al. & Logistics capability & $\begin{array}{l}\text { Supply chain uncer- } \\
\text { tainty and risk }\end{array}$ & $\begin{array}{l}\text { Logistics perfor- } \\
\text { mance }\end{array}$ & PLS-SEM & $\begin{array}{l}\text { Australian courier } \\
\text { firms }\end{array}$ \\
\hline 2018 & Jadhav et al. & $\begin{array}{l}\text { Supply chain orien- } \\
\text { tation }\end{array}$ & $\begin{array}{l}\text { Organization's inter- } \\
\text { nal supply chain sus- } \\
\text { tainability practices }\end{array}$ & $\begin{array}{l}\text { Supply chain envi- } \\
\text { ronmental sustain- } \\
\text { ability performance }\end{array}$ & SEM & $\begin{array}{l}\text { Australian manufac- } \\
\text { turing firms }\end{array}$ \\
\hline 2018 & Lo et al. & $\begin{array}{l}\text { Top management } \\
\text { support }\end{array}$ & $\begin{array}{l}\text { Supplier develop- } \\
\text { ment }\end{array}$ & $\begin{array}{l}\text { Upstream green } \\
\text { supply chain inte- } \\
\text { gration }\end{array}$ & SEM & International firms \\
\hline 2018 & Agarwal et al. & Market pressures & Internal impetus & $\begin{array}{l}\text { Green Supply } \\
\text { Chain Manage- } \\
\text { ment adoption }\end{array}$ & $\begin{array}{l}\text { Partial } \\
\text { Least } \\
\text { Squares } \\
\text { (PLS) }\end{array}$ & $\begin{array}{l}\text { USA manufacturing } \\
\text { companies }\end{array}$ \\
\hline 2019 & $\begin{array}{l}\text { Khan and Wis- } \\
\text { ner }\end{array}$ & $\begin{array}{l}\text { Supply chain inte- } \\
\text { gration }\end{array}$ & Supply chain agility & $\begin{array}{l}\text { Firm } \\
\text { performance }\end{array}$ & SEM & $\begin{array}{l}\text { Pakistan public- } \\
\text { owned companies }\end{array}$ \\
\hline 2019 & Porter & $\begin{array}{l}\text { Supply chain inte- } \\
\text { gration }\end{array}$ & $\begin{array}{l}\text { Organizational cul- } \\
\text { ture }\end{array}$ & Firm performance & $\begin{array}{l}\text { Multiple re- } \\
\text { gression }\end{array}$ & $\begin{array}{l}\text { USA supply chain } \\
\text { professionals and } \\
\text { procurement special- } \\
\text { ists }\end{array}$ \\
\hline
\end{tabular}

\section{THEORETICAL MODELS WITH MODERATED MEDIATION DE- SIGNS AND TESTS}

Moderated mediation, also known as conditional indirect effects, occurs when the treatment effect of an independent variable $A$ on an outcome variable $C$ via a mediator variable $\mathrm{B}$ differs depending on the levels of a moderator variable D. Specifically, either the effect of A on B, and/or the effect of B on C depends on the level of D (Preacher et al., 2007). To test for moderated mediation, some recommend examining a series of models, sometimes called a piecemeal approach, and looking at the overall pattern of results (Muller et al., 2005). This approach is similar to the previously mentioned Baron and Kenny method for testing mediation by analyzing a series of three regressions (Baron \& Kenny, 1986). They suggest a single overall test would be insufficient to analyze the complex processes at play in moderated mediation, and would not allow one to differentiate between moderated mediation and mediated moderation. In terms of the method of the moderated mediation model, bootstrapping has also been suggested as a method of estimating the sampling distributions of a moderated mediation model to generate confidence intervals (Preacher et al., 2007). This method has the advantage of not requiring any assumptions be made about the shape of the sampling distribution. Preacher et al. (2007) 
created an SPSS macro that provides bootstrapping estimations as well as Johnson-Neyman results. Their macro was made obsolete with the release of PROCESS for SPSS and SAS.

PROCESS is an observed variable ordinary least squares (OLS) and logistic regression path analysis modeling tool. It is widely used in social, business, and health sciences for estimating direct and indirect effects in single and multiple mediator models (parallel and serial), two and three way interactions in moderation models along with simple slopes and regions of significance for probing interactions, and conditional indirect effects in moderated mediation models with a single or multiple mediators or moderators (Hayes, 2013a). PROCESS generates direct and indirect effects in mediation and mediated moderation models, conditional effects in moderation models, and conditional indirect effects in moderated mediation models with a single or multiple mediator. PROCESS offers various tools for probing 2 and 3 way interactions and can construct percentile based bootstrap confidence intervals for conditional and unconditional indirect effects. In mediation models, multiple mediator variables can be specified to operate in parallel or in sequence. Heteroscedasticityconsistent standard errors are available for inference about paths coefficients, in the Sobel test for indirect effects, and when probing interactions in moderation analysis. Various measures of effect size for indirect effects are generated in mediation models, along with bootstrap confidence intervals for effect size inference. An option is available for patriating out contextual level variation when individual data are nested under a higher-level organizational structure. Individual paths in moderated mediation models can be estimated as moderated by one or two variables either additively or multiplicatively (Hayes, 2013b). Recently, it was found that either the effect of the independent variable on the mediator variable, and/or the effect of the mediator variable on the outcome var- iable depends on the level of the moderator variable (Drummond et al., 2016). In addition, Cheung and Lau (2017) extended the latent moderated structural equations (LMS) method-which corrects for measurement errors when estimating latent interaction effects - to the study of the moderated mediation of latent variables. Simulations were conducted to compare the regression approach and the LMS approach. On the other hand, moderated mediation analyses using Bayesian methods are alternatives to investigating theoretical models of moderated mediation (Wang \& Preacher, 2015).

Table 3 shows regression analyses and SEM using PROCESS version 3 are the main methods used to examine a moderated mediation model for Internet user behavior. In terms of subject background, we examined international manufacturing companies, global firms, USA sellers, Chinese manufacturing firms, USA soft drink industry firms, USA manufacturing and service subjects, China food manufacturing firms and Ghana firms etc. In terms of moderated mediator variables, we found the following designs and tests: global sourcing/sustainable supply chain management, supply chain planning/capabilities, trade offers expected by the sellers/aggregation of seller's cost information, knowledge acquisition, knowledge combination/servitisation, forecast bias/vertical integration, operational integration/upstream and downstream partners, collaborative culture/inter-organizational systems, supply chain organizational learning/uncertainty, Environmental dynamism/ Operating and marketing capabilities and Flexibility capability/ Ownership structure etc.. Supply chain integration, collaboration and performance are main considerations in terms of the designs and tests of theoretical models of moderated mediation.

Table 3 Theoretical models with moderated mediation designs and tests

\begin{tabular}{|c|c|c|c|c|c|c|}
\hline Year & Author(s) & $\begin{array}{l}\text { Independent varia- } \\
\text { ble }\end{array}$ & Moderated mediation & $\begin{array}{l}\text { Dependent varia- } \\
\text { ble }\end{array}$ & Method & Subjects \\
\hline 2014 & $\begin{array}{l}\text { Gualandris } \\
\text { et al. }\end{array}$ & Social responsibility & $\begin{array}{l}\text { Global sourcing/ Sus- } \\
\text { tainable supply chain } \\
\text { management }\end{array}$ & $\begin{array}{l}\text { Ecological effi- } \\
\text { ciency }\end{array}$ & SEM & $\begin{array}{l}\text { International manufac- } \\
\text { turing companies }\end{array}$ \\
\hline 2015 & $\begin{array}{l}\text { Srinivasan } \\
\& \text { Swink }\end{array}$ & $\begin{array}{l}\text { Supply chain inte- } \\
\text { gration activities }\end{array}$ & $\begin{array}{l}\text { Supply chain planning/ } \\
\text { Capabilities }\end{array}$ & $\begin{array}{l}\text { Operational perfor- } \\
\text { mance }\end{array}$ & SEM & Global firms \\
\hline 2016 & $\begin{array}{l}\text { Miller \& } \\
\text { Drake }\end{array}$ & $\begin{array}{l}\text { Information asym- } \\
\text { metry }\end{array}$ & $\begin{array}{l}\text { Trade offers expected } \\
\text { by the sellers/ Aggre- } \\
\text { gation of seller's cost } \\
\text { information }\end{array}$ & $\begin{array}{l}\text { Relation-specific } \\
\text { investment }\end{array}$ & SEM & USA sellers \\
\hline 2017 & Zhang et al. & $\begin{array}{l}\text { Social capital ef- } \\
\text { fects }\end{array}$ & $\begin{array}{l}\text { Knowledge acquisi- } \\
\text { tion; Knowledge com- } \\
\text { bination/Servitisation }\end{array}$ & $\begin{array}{l}\text { Operational perfor- } \\
\text { mance }\end{array}$ & SEM & $\begin{array}{l}\text { China manufacturing } \\
\text { firms }\end{array}$ \\
\hline 2017 & Wan et al. & Product variety & $\begin{array}{l}\text { Forecast bias/ Vertical } \\
\text { integration }\end{array}$ & Inventory level & $\begin{array}{l}\text { General- } \\
\text { ized esti- } \\
\text { mating } \\
\text { equation }\end{array}$ & $\begin{array}{l}\text { USA soft drink industry } \\
\text { firms }\end{array}$ \\
\hline 2017 & $\begin{array}{l}\text { Vanpoucke } \\
\text { et al. }\end{array}$ & $\begin{array}{l}\text { Information ex- } \\
\text { change }\end{array}$ & $\begin{array}{l}\text { Operational integra- } \\
\text { tion/ Upstream and } \\
\text { downstream partners }\end{array}$ & $\begin{array}{l}\text { Cost-efficiency; } \\
\text { Delivery perfor- } \\
\text { mance; Process } \\
\text { flexibility }\end{array}$ & $\begin{array}{l}\text { Con- } \\
\text { strained } \\
\text { non-linear } \\
\text { regression } \\
\text { (CNLR) }\end{array}$ & $\begin{array}{l}\text { International manufac- } \\
\text { turing survey }\end{array}$ \\
\hline
\end{tabular}


Table 3 (con`t) Theoretical models with moderated mediation designs and tests

\begin{tabular}{|l|l|l|l|l|l|l|l|}
\hline 2018 & Zhang et al. & IOS appropriation & $\begin{array}{l}\text { Collaborative culture/ } \\
\text { Inter-organizational } \\
\text { systems }\end{array}$ & $\begin{array}{l}\text { supply chain col- } \\
\text { laboration }\end{array}$ & SEM & $\begin{array}{l}\text { USA manufacturing } \\
\text { firms }\end{array}$ \\
\hline 2018 & Ojha et al. & $\begin{array}{l}\text { Transformational } \\
\text { leadership }\end{array}$ & $\begin{array}{l}\text { Supply chain organiza- } \\
\text { tional learning/ Uncer- } \\
\text { tainty }\end{array}$ & $\begin{array}{l}\text { Supply chain ambi- } \\
\text { dexterity }\end{array}$ & $\begin{array}{l}\text { SEM } \\
\text { anA manufacturing } \\
\text { and service subjects }\end{array}$ \\
\hline 2019 & $\begin{array}{l}\text { Song and } \\
\text { Yang }\end{array}$ & $\begin{array}{l}\text { Food traceability- } \\
\text { core capabilities }\end{array}$ & $\begin{array}{l}\text { Environmental dyna- } \\
\text { mism/ Operating and } \\
\text { marketing capabilities }\end{array}$ & $\begin{array}{l}\text { Food traceability- } \\
\text { performance }\end{array}$ & SEM & $\begin{array}{l}\text { China food manufac- } \\
\text { turing firms }\end{array}$ \\
\hline $\begin{array}{l}\text { Amoako- } \\
\text { Gyampah et } \\
\text { al. }\end{array}$ & $\begin{array}{l}\text { Supplier relationship } \\
\text { management }\end{array}$ & $\begin{array}{l}\text { Flexibility capability/ } \\
\text { Ownership structure }\end{array}$ & Firm performance & $\begin{array}{l}\text { Regres- } \\
\text { sion }\end{array}$ & Ghana firms \\
\hline
\end{tabular}

\section{THEORETICAL MODELS WITH MEDIATED MODERATION DE- SIGNS AND TESTS}

In mediated moderation, the main difference between the moderated mediation and mediated moderation processes is whether there is overall moderation of the treatment effect of A on outcome variable $\mathrm{C}$. If there is, then there is mediated moderation. If there is no overall moderation of $\mathrm{A}$ on $\mathrm{C}$, then there is moderated mediation (Muller et al., 2005). Bucy and Tao (2007) propose a mediated moderation model and maintain that interactivity, serving as a media stimulus, should be defined in terms of intrinsic media attributes. They point out that interactivity in a mediated moderation model, which resides in media technology, serves as the manipulated independent variable, while perceived interactivity, which consists of user perceptions, functions to transform the impact of interactivity. Importantly, the objective existence of interactive attributes does not guarantee the subjective experience of interactivity - but actual technology use may. Both interactivity and perceived interactivity thus play an important role in theorizing the effects of information technology yet represent disparate constructs and should be treated as having independent influences. Thus, in the mediated moderation model, the interaction effects of the independent and moderator variables on the dependent variable are transmitted through the mediator variable. A prerequisite of mediated moderation is the occurrence of overall moderation between the independent and dependent variables (Baron \& Kenny, 1986). The effect of the independent variable on the dependent variable must depend on the moderator variable. There are at least three different types of mediated moderation: between the independent and mediator variables, between the mediator and dependent variables, or both (Muller et al., 2005). Mediated moderation can be used to explain the causal relationship between four variables. Therefore, the mediated moderation model should occur between interactivity and perceived interactivity. The model accommodates the possibility that the same interactive features may produce different levels of interactivity design among different researchers depending on theoretical model differences (Holbert, 2005). Based on research findings, few mediated moderation models have been published in academic journals because of difficulties not only in sample analysis and testing, but also in hypotheses and theoretical model development for specific problem domains and subjects.

Table 4 shows three articles concerning behavior studies of Internet users with mediated moderation using hierarchical multiple regression and SEM analyses. USA information technology firms, Taiwanese industrial buyers and North American firms were research subjects. Mediated moderator variables included governance-knowledge fit/IT governance, attribution/industrial brand equity and forecast accuracy/internal integration, forecasting process quality, effective use of advanced systems, valuation of forecasting, etc.

Table 4 Theoretical models with mediated moderation designs and tests

\begin{tabular}{|l|l|l|l|l|l|l|}
\hline Year & Author(s) & $\begin{array}{l}\text { Independent var- } \\
\text { iable }\end{array}$ & \multicolumn{1}{|c|}{ Mediated moderation } & Dependent variable & Method & Subjects \\
\hline 2014 & Xue & $\begin{array}{l}\text { Strategic risk tak- } \\
\text { ing }\end{array}$ & $\begin{array}{l}\text { Governance-knowledge } \\
\text { fit/IT governance }\end{array}$ & $\begin{array}{l}\text { Supply chain digitiza- } \\
\text { tion }\end{array}$ & $\begin{array}{l}\text { SEM } \\
\text { USA information } \\
\text { technology firms }\end{array}$ \\
\hline 2015 & Pai et al. & $\begin{array}{l}\text { Corporate Social } \\
\text { Responsibility }\end{array}$ & $\begin{array}{l}\text { Attribution/ Industrial brand } \\
\text { equity }\end{array}$ & Brand Advocacy & $\begin{array}{l}\text { Hierarchical } \\
\text { multiple re- } \\
\text { gression }\end{array}$ & $\begin{array}{l}\text { Taiwan industrial } \\
\text { buyers }\end{array}$ \\
\hline Suresh & $\begin{array}{l}\text { Cost reduction; } \\
\text { Delivery perfor- } \\
\text { mance }\end{array}$ & $\begin{array}{l}\text { Forecast accuracy/ Internal } \\
\text { integration; Forecasting } \\
\text { process quality; Effective } \\
\text { use of advanced systems; } \\
\text { Evaluation of forecasting }\end{array}$ & $\begin{array}{l}\text { Forecasting manage- } \\
\text { ment competence }\end{array}$ & PLS-SEM & $\begin{array}{l}\text { North America } \\
\text { firms }\end{array}$ \\
\hline
\end{tabular}

Accordingly, the theoretical models with moderators, mediators, moderated mediation and mediated moderation described in the above context provide examples not only in their academic theory but also in the development of the study of supply chain management study. In doing so, this study offers deductive and inductive reviews of relevant supply 
chain management research issues to readers for future studies. In addition, this study proposes some theoretical discussions on in next section.

\section{DISCUSSIONS}

\subsection{Supply Chain Management Research Devel- opment}

The research development of SCM has mainly gone through four phases of history. The first phase was the independent logistics distribution and logistics cost management phase, which mainly studied physical distribution and distribution systems to downstream manufacturers. In this phase, supply chain management was inseparable from the development of manufacturing automation, the evolution of business management and the evolution of enterprise information systems. In the 1950s and 1960s, manufacturers emphasized large-scale production to reduce unit production costs, that is, operational strategies for mass production. At that time, the production of enterprises considered market factors less. This phase was also characterized by a lack of flexibility in production and manufacturing, the slow development of new products, and the almost total reliance on the internal technology and capabilities of the enterprise. Therefore, the operational bottleneck of the enterprise was solved by increasing the inventory, and the cooperation and development between enterprises were rarely considered. Purchasing at the time was only considered a support activity for production, and managers were less concerned with procurement activities. In the 1970s, a manufacturing resource plan was introduced, and managers realized that the amount of inventory had a significant impact on manufacturing costs, new product development, and production lead times. Thus, business performance was improvement by shifting to new material management.

The second phase was the integrated logistics management phase, focusing on the integration of intra-enterprise logistics and external logistics, and researching inter-enterprise procurement and supply strategies, emphasizing the strengthening of cooperation. After the 1980s, global competition intensified. Some large multinational companies faced market competition and maintained their leading position by providing low-cost, high-quality, reliable products and more flexible designs. Manufacturing companies began to introduce JIT productivity concepts, and Japanese companies implemented JIT to improve manufacturing efficiency, shorten production cycles and reduce inventory. Manufacturers were aware of the importance of strategic partnerships as JIT mitigated production and scheduling problems through a fast-paced manufacturing environment and low inventory. Therefore, when manufacturers and suppliers began to develop strategic supply relationships, the concept of supply chain management emerged. In this phase, the specialization of procurement, logistics and transportation processes promoted the further development of material management concepts. Manufacturing Resource Planning (MRP II) emphasizes the integration of functions and resources within an enterprise, and the integration of internal resource planning requires the cooperation of external suppliers and distributors. Manufacturing companies integrated internal and external logistics systems, which leads to the concept of integrated logistics.
The third phase was the integration of the supply chain management phase, which focused on the overall supply chain research from the supplier's supplier to the customer's customer, focusing on the overall value chain efficiency and value added. After the 1990s, supply chain management continued to grow and the supply chain expanded into an overall value chain of suppliers, manufacturers, distributors and customers. The efficiency of procurement and supply required more consideration of coordination between cost and quality. Manufacturers eliminated non-value-added activities by purchasing raw materials from selected suppliers or certified suppliers, such as raw material quality inspections, warehousing inspections, etc. Many manufacturers and retailers worked closely together to increase the efficiency of crossenterprise value chains. For example, in the development of new products, manufacturers' integrated suppliers and customers, used the research and development capabilities and technology of partners, improved the research and development cycle, and enhanced core competitiveness. Distributors and retailers seamlessly connected their distribution and transportation providers to achieve direct delivery and eliminate value-added activities such as item inspections (Uvet, 2020).

The fourth phase, after the 20th century, was the development of information technology and decision support systems. Information technology is a key factor in enabling effective supply chain management. In this phase, the basic question of supply chain management is what data should be passed and how it is analyzed and utilized in terms of integration and collaboration. The impact of the Internet, the role of e-commerce, information technology and decision support systems have become the main tools for companies to gain market competitive advantage. On the other hand, customer value is a measure of how much a supply chain or firm contributes to its customers in terms of market performance. This metric is measured by all the goods, services, and intangibles that the company provides. This indicator has replaced indicators such as quality and customer satisfaction in recent years.

\subsection{Trends in Supply Chain Management Re- search}

In regard to time and speed, in this study, the supply chain environment, time and speed have been regarded as the main sources of improving the competitive advantage of enterprises. The drag of one link often affects the operation of the entire supply chain. Each enterprise in the supply chain realizes the close connection between logistics and information flow through various means to achieve the quick response to the final customer requirements, reduce the inventory cost, and improve the overall competition level of the supply chain in electronic commerce (E-commerce) (Das et al., 2020).

In regard to quality and asset productivity, in this study, supply chain management involves many links, and it needs to be closely linked and ensure the quality of each link. Any link, such as the quality of transportation services, will directly affect the quantity of suppliers' stocking and the number of distributors' warehousing, which will ultimately affect users' evaluation of product quality, timeliness and price. 
Nowadays, more and more companies believe that logistics quality innovation is evolving into a powerful force to improve supply chain performance. On the other hand, manufacturers are increasingly concerned about asset productivity. Improving asset productivity is not just about reducing inventory within the company, but more importantly, reducing inventory in the supply chain. The trends in supply chain management require companies to collaborate and share data to reduce inventory across the supply chain.

In regard to organizational streamlining, in this study, the type and number of members in the supply chain are the direct cause of the complexity of supply chain management. Under the current supply chain development trend, more and more companies are beginning to consider reducing the number of logistics providers, and this trend is very obvious and rapid. For example, multinational clients are more willing to outsource their global logistics supply chain to a few partners, ideally logistics providers. This is not only beneficial to management, but also helps to provide uniform standard services on a global scale, and better demonstrates the overall advantages of global supply chain management.

In regard to customer service, in this study, more and more supply chain members are beginning to value customer service and customer satisfaction. Supply chain management needs to pay more attention to customers' feelings about the service level, and the measurement of service level is also based on customer satisfaction. The result of the shift in the focus of customer service is to attach importance to the relationship with logistics firms, and regard logistics firms as partners in providing a high level of service.

\subsection{Development of Theoretical Models of Supply Chain Management}

A theoretical model is a representation of a problem domain using general rules and concepts; also it is a simplified and idealized understanding of solution alternatives (Wieteska, 2020). This study examines the supply chain management research issue by reviewing the development SCM theoretical models and illustrating theoretical model types with moderators, mediators, moderated mediation and mediated moderation variables. The ninety-seven articles reviewed here compiled the variables, research methods, and research subjects theoretical models of supply chain management research from 2014-2019. The theoretical models with moderators and mediators are mature theoretical models in SCM research. In recent years, theoretical models with moderated mediation have been an emerging development, applied in the study of this research issue. In addition, with the rapid development of statistical and mathematical analysis tools and the pursuit of excellence in science and social science theories, theoretical models with mediated moderation are destined develop further in the future of SCM research.

\section{CONCLUSION}

This research is the first article in terms of investigating supply chain management studies using a theoretical model review based on a qualitative historical review research in supply chain issue. This survey only provides a static picture of a very dynamic area, but several implications can be drawn from the supply chain management literature. Different reviews and survey methods must be implemented to broaden the knowledge base. This study also contributes to the field by providing past, current and future research directions with potential understandings and matters of significance. Finally, even if the which articles related to theoretical models of supply chain management published in various journals have captured the attention of several research communities and categories, very few multidisciplinary studies seem to have been conducted on the subject. Therefore, this study provides a valuable reference for future studies of supply chain management.

\section{REFERENCES}

Acerbi, F., \& Taisch, M. (2020). A literature review on circular economy adoption in the manufacturing sector. Journal of Cleaner Production, 10, 123086. https://doi.org/10.1016/j.jclepro.2020.123086

Agrawal, A., Muthulingam, S., \& Rajapakshe, T. (2017). How Sourcing of Interdependent Components Affects Quality in Automotive Supply Chains. Production and Operations Management, 26(8), pp.1512-1533.

Agarwal, A., Giraud-Carrier, C., \& Li, Y. (2018). A mediation model of green supply chain management adoption: The role of internal impetus. International Journal of Production Economics, 205(3), pp.342-358.

Al Humdan, E., Shi, Y., \& Behnia, M. (2020). Supply chain agility: a systematic review of definitions, enablers and performance implications. International Journal of Physical Distribution \& Logistics Management, 50(2), pp.287-312.

Ambulkar, S., Blackhurst, J., \& Grawe, S. (2015). Firm's resilience to supply chain disruptions: Scale development and empirical examination. Journal of Operations Management, 33-34, pp.111-122.

Amoako-Gyampah, K., Boakye, K. G., Adaku, E., \& Famiyeh, S. (2019). Supplier relationship management and firm performance in developing economies: A moderated mediation analysis of flexibility capability and ownership structure. International Journal of Production Economics, 208, PP.160-170.

Asghari, M., \& Al-e-hashem, S.M.J.M. (2020). Green vehicle routing problem: A state-of-the-art review. International Journal of Production Economics, 107899, In Press, https://doi.org/10.1016/j.ijpe.2020.107899

Baron, R. M., \& Kenny, D. A. (1986). The moderator-mediator variable distinction in social psychological research: Conceptual, strategic and statistical considerations. Journal of Personality and Social Psychology, 51, 1173-1182.

Basnet, C., \& Seuring, S. (2016). Demand-oriented Supply Chain Strategies - A Review of the Literature. Operations and Supply Chain Management, 9(2), pp.73-89.

Batista, L., Bourlakis, M., Smart, P., \& Maull, R. (2018). In search of a circular supply chain archetype - a contentanalysis-based literature review. Production Planning \& Control, 29(6), pp.438-451.

Bode, C., \& Macdonald, R. (2016). Stages of Supply Chain Disruption Response: Direct, Constraining, and Mediating Factors for Impact Mitigation. Decision Sciences, 48(5), pp.836-874.

Bonn, A., Cronin, Jr. J., \& Cho, M. (2015). Do Environmental Sustainable Practices of Organic Wine Suppliers Affect Consumers' Behavioral Intentions? The Moderating 
Role of Trust. Cornell Hospitality Quarterly, 57(1), pp.21-37

Brandon-Jones, E., Squire, B., Autry, W., \& Petersen, J. (2014). A Contingent Resource-Based Perspective of Supply Chain Resilience and Robustness. Journal of Supply Chain Management, 50(3), pp.55-73.

Brandon-Jones, E., Squire, B., \& Van Rossenberg, G. T. (2015). The impact of supply base complexity on disruptions and performance: the moderating effects of slack and visibility. International Journal of Production Research, 53(22): 6903-6918.

Brinkhoff, A., Özer, Ö., \& Sargut, G. (2014). All You Need Is Trust? An Examination of Inter-organizational Supply Chain Projects. Production and Operations Management, 24(2), pp.181-200.

Bucy, E.P., \& Tao, C.C. (2007). The mediated moderation model of interactivity. Media Psychology, 9(3), pp.647672.

Busse, C. (2015). Doing Well by Doing Good? The Self-interest of Buying Firms and Sustainable Supply Chain Management. Journal of Supply Chain Management, 52(2), pp.28-47.

Caniato, F., \& Größler, A. (2015). The moderating effect of product complexity on new product development and supply chain management integration. Production Planning \& Control, 26(16), pp.1306-1317.

Chan, T. L., Ngai, W. T., \& Moon, K. L. (2017). The effects of strategic and manufacturing flexibilities and supply chain agility on firm performance in the fashion industry. European Journal of Operational Research, 259(2), pp.486-499.

Chang, W., Ellinger, E., Kim, K., \& Franke, R. (2016). Supply chain integration and firm financial performance: A meta-analysis of positional advantage mediation and moderating factors. European Management Journal, 34(3), pp.282-295.

Chang, H. H., Wong, K. H., \& Chiu, W. S. (2019). The effects of business systems leveraging on supply chain performance: Process innovation and uncertainty as moderators. Information \& Management, 56(6), p.103140.

Chen, Y., Wu, Y., \& Wu, T. (2015). Moderating effect of environmental supply chain collaboration: Evidence from Taiwan. International Journal of Physical Distribution \& Logistics Management, 45(9/10), pp.959-978.

Cheung, G. W., \& Lau, R. S. (2017). Accuracy of Parameter Estimates and Confidence Intervals in Moderated Mediation Models. Organizational Research Methods, 20(4), pp.746-769.

Christopher, M. (1998). Logistics and Supply Change Management. Pitman, ed. 2.

David, D., \& Sava, F.A. (2015). Designs for Studying Mediation. John Wiley \& Sons, Inc, New York.

Das, D., Kumar, R., \& Rajak, M.K. (2020). Designing a Reverse Logistics Network for an E-Commerce Firm: A Case Study. Operations and Supply Chain Management, 13(1), pp.48-63.

Ding, L., Lam, K. S., Cheng, T. C. E., \& Zhou, H. (2018). A review of short-term event studies in operations and supply chain management. International Journal of Production Economics, 200, pp.329-342.
Doering, T., \& Suresh, C. (2016). Forecasting and Performance: Conceptualizing Forecasting Management Competence as a Higher-Order Construct. Journal of Supply Chain Management, 52(4), pp.77-91.

Drummond, S., O’Driscoll, P., Brough, P., Kalliath, T., Siu, O.L., Timms, C., Riley, D., \& Lo, D. (2016). The relationship of social support with well-being outcomes via work-family conflict: Moderating effects of gender, dependents and nationality. Human Relations, 70, pp. 544565.

Dubey, R., Gunasekaran, A., Childe, J., Papadopoulos, T., \& Helo, P. (2018). Supplier relationship management for circular economy: Influence of external pressures and top management commitment. Management Decision, 57(4), pp. 767-790

Durach, F., Glasen, C., \& Straube, F. (2017). Disruption causes and disruption management in supply chains with Chinese suppliers: Managing cultural differences. International Journal of Physical Distribution \& Logistics Management, 47(9), pp.843-863.

Ebrahimi, S. M., Koh, S.C.L., Genovese, A., \& Kumar, N. (2018). Structure-integration relationships in oil and gas supply chains. International Journal of Operations \& Production Management, 38(2), pp.424-445.

Eckstein, D., Goellner, M., Blome, C., \& Henke, M. (2015). The performance impact of supply chain agility and supply chain adaptability: the moderating effect of product complexity. International Journal of Production Research, 25(10), pp.3028-3046.

Edwards, J. R. (2002). Alternatives to difference scores: Polynomial regression analysis and response surface methodology. In F. Drasgow \& N. W. Schmitt (Eds.), Advances in measurement and data analysis (pp. 350-400). Jossey-Bass, San Francisco.

El-Kassar, A. N., \& Singh, S. K. (2018). Green innovation and organizational performance: The influence of big data and the moderating role of management commitment and HR practices. Technological Forecasting and Social Change, 144, pp. 483-498

Flynn, B., Koufteros, X., \& Lu, G. (2016). On Theory in Supply Chain Uncertainty and its Implications for Supply Chain Integration. Journal of Supply Chain Management, 52(3), pp. 3-27.

Gandhi, A. V., Shaikh, A., \& Sheorey, P. A. (2017). Impact of supply chain management practices on firm performance: Empirical evidence from a developing country. International Journal of Retail \& Distribution Management, 45(4), pp. 366-384.

Giunipero, L. C., Hooker, R., Joseph-Matthews, S., Yoon, T. E., \& Brudvig, S. (2008). A Decade of SCM Literature: Past, Present and Future Implications. Journal of Supply Chain Management, 44(4), pp. 66-86.

Gligor, D. (2017). Re-Examining Supply Chain Fit: An Assessment of Moderating Factors. Journal of Business Logistics, 38(4), pp. 253-265.

Gokarn, S., \& Kuthambalayan, T. S. (2019). Creating sustainable fresh produce supply chains by managing uncertainties. Journal of Cleaner Production, 207, pp.908919. 
Gómez-Cedeño, M., Castán-Farrero, J. M., Guitart-Tarrés, L., \& Matute-Vallejo, J. (2015). Impact of human resources on supply chain management and performance. Industrial Management \& Data Systems, 115(1), pp. 129-157.

Gonzalez-Loureiro, M., Dabic, M., \& Kiessling, T. (2015). Supply chain management as the key to a firm's strategy in the global marketplace: Trends and research agenda. International Journal of Physical Distribution \& Logistics Management, 45(1/2), pp. 159-181.

González-Teruel, A., \& Pérez-Pulido, M. (2020). The diffusion and influence of theoretical models of information behaviour. The case of Savolainen's ELIS model. Journal of Documentation, 76(5), pp.1069-1089.

Gualandris, J., Golini, R., \& Kalchschmidt, M. (2014). Do supply management and global sourcing matter for firm sustainability performance?: An international study. Supply Chain Management: An International Journal, 19(3), pp. 258-274.

Hayes. A.F. (2013a). Introduction to mediation, moderation, and conditional process analysis: A regression based approach. The Guilford Press, New York.

Hayes, A.F. (2013b). SPSS PROCESS documentation. Available at 2 January 2013, http://www.marketingwiwi.uni-jena.de/wmarmedia/dokumente/WS+15 16/DAM/Process_Anleitung alle Modelle.pdf

Holbert, R. L. (2005). Debate viewing as mediator and partisan reinforcement in the relationship between news use and vote choice. Journal of Communication, 55(1), pp. $85-102$.

Huo, B., Zhang, C., \& Zhao, X. (2015). The effect of IT and relationship commitment on supply chain coordination: A contingency and configuration approach. Information \& Management, 52(6), pp. 728-740.

Jadhav, A., Orr, S., \& Malik, M. (2018). The role of supply chain orientation in achieving supply chain sustainability. International Journal of Production Economics, https://doi.org/10.1016/j.ijpe.2018.07.031

Jasti, N. V. K., \& Kodali, R. (2015). Lean production: literature review and trends. International Journal of Production Research, 53(3), pp.867-885.

Kabra, G., Ramesh, A., Akhtar, P., \& Dash, M. K. (2017). Understanding behavioural intention to use information technology: Insights from humanitarian practitioners. Telematics and Informatics, 34(7), pp.1250-1261.

Kache, F., \& Seuring, S. (2014). Linking collaboration and integration to risk and performance in supply chains via a review of literature reviews. Supply Chain Management: An International Journal, 19(5/6), pp.664-682.

Kaufmann, L., \& Gaeckler, J. (2015). A structured review of partial least squares in supply chain management research. Journal of Purchasing and Supply Management, 21(4), pp.259-272.

Kembro, J., Selviaridis, K., \& Näslund, D. (2014). Theoretical perspectives on information sharing in supply chains: a systematic literature review and conceptual framework. Supply Chain Management: An International Journal, 19(5/6), pp.609-625.

Kenny, D.A., Kashy, D.A., \& Cook, W.L. (2006). Dyadic Data Analysis. The Guilford Press, New York.
Khalid, R. U., Seuring, S., Beske, P., Land, A., Yawar, S. A. \& Wagner, R. (2015). Putting sustainable supply chain management into base of the pyramid research. Supply Chain Management: An International Journal, 20(6), pp.681-696.

Khan, H., \& Wisner, J.D. (2019). Supply Chain Integration, Learning, and Agility: Effects on Performance. Operations and Supply Chain Management, 12(1), pp.14-23.

Kim, M., Hwang, Y. \& Rho, J. J. (2016). The impact of RFID utilization and supply chain information sharing on supply chain performance: Focusing on the moderating role of supply chain culture. Maritime Economics \& Logistics, 18(1), pp.78-100.

Kline, R. (2015). Principles and Practice of Structural Equation Modeling (Fourth ed.). The Guilford Press, New York.

Kumar, G., Banerjee, R. N., Meena, P. L., \& Ganguly, K. (2016). Collaborative culture and relationship strength roles in collaborative relationships: a supply chain perspective. Journal of Business \& Industrial Marketing, 31(5), pp.587-599.

Ledwoch, A., Yasarcan, H., \& Brintrup, A. (2018). The moderating impact of supply network topology on the effectiveness of risk management. International Journal of Production Economics, 197, pp.13-26.

Lee, H. Y., Seo, Y. J., \& Dinwoodie, J. (2016). Supply chain integration and logistics performance: the role of supply chain dynamism. The International Journal of Logistics Management, 27(3), pp.668-685.

Levi-Bliech, M., Naveh, G., Pliskin, N., \& Fink, L. (2018). Mobile Technology and Business Process Performance: The Mediating Role of Collaborative Supply-Chain Capabilities. Information Systems Management, 35(4), pp.308-329.

Li, Y. H., \& Huang, J. W. (2017). The moderating role of relational bonding in green supply chain practices and performance. Journal of Purchasing and Supply Management, 23(4), pp.290-299.

Li, E. L., Zhou, L., \& Wu, A. (2017). The supply-side of environmental sustainability and export performance: The role of knowledge integration and international buyer involvement. International Business Review, 26(4), pp.724-735.

Liao, S. H., \& Kuo, F. I. (2014). The study of relationships between the collaboration for supply chain, supply chain capabilities and firm performance: A case of the Taiwan's TFT-LCD industry. International Journal of Production Economics, 156, pp.295-304.

Liao, S. H., Hu, D. C., \& Ding, L. W. (2017). Assessing the influence of supply chain collaboration value innovation, supply chain capability and competitive advantage in Taiwan's networking communication industry. International Journal of Production Economics, 191, 143153.

Liu, Z., Prajogo, D., \& Oke, A. (2016). Supply Chain Technologies: Linking Adoption, Utilization, and Performance. Journal of Supply Chain Management, 52(4), pp.22-41.

Liu, Y., Zhu, Q., \& Seuring, S. (2017). Linking capabilities to green operations strategies: The moderating role of corporate environmental proactivity. International Journal of Production Economics, 187, pp.182-195. 
Lo, M., Zhang, S., Wang, Z., \& Zhao, X. (2018). The impact of relationship quality and supplier development on green supply chain integration: A mediation and moderation analysis. Journal of Cleaner Production, 202, pp.524-535.

Luo, J., Chong, Y. L., Ngai, W. T., \& Liu, J. (2015). Reprint of "Green Supply Chain Collaboration implementation in China: The mediating role of guanxi. Transportation Research Part E: Logistics and Transportation Review, 74, pp.37-49.

Lu, S. H., Cantor, D., \& Hofer, C. (2018). Content analysis in SCM research: past uses and future research opportunities. The International Journal of Logistics Management, 29(1), pp.152-190.

Maestrini, V., Luzzini, D., Maccarrone, P., \& Caniato, F. (2017). Supply chain performance measurement systems: A systematic review and research agenda. International Journal of Production Economics, 183, pp.299315.

Mandal, S. (2018). Exploring the influence of big data analytics management capabilities on sustainable tourism supply chain performance: the moderating role of technology orientation. Journal of Travel \& Tourism Marketing, 35(8), pp.1104-1118.

Mariadoss, B. J., Chi, T., Tansuhaj, P., \& Pomirleanu, N. (2016). Influences of Firm Orientations on Sustainable Supply Chain Management. Journal of Business Research, 69(9), pp.3406-3414.

Miller, F., \& Drake, A. (2016). Using information asymmetry to mitigate hold-ups in supply chains. Management Accounting Research, 32, pp.16-26.

Mokadem, M. (2016). ISO 9000 moderation role over supply chain alignment in manufacturing context. Journal of Manufacturing Technology Management, 27(3), pp.338-363.

Mueller, R.O. (1996). Basic Principles of structural equation modeling: An introduction to LISREL and EQS. Springer-Verlag, New York.

Mujkic, Z., Qorri, A., \& Kraslawski, A. (2018). Sustainability and Optimization of Supply Chains: a Literature Review. Operations and Supply Chain Management, 11(4), pp.186-199.

Muller, D., Judd, C.M., \& Yzerbyt, V. (2005). When Moderation is Mediated and Mediation is Moderated. Journal of Personality and Social Psychology, 89(6), pp.852-63.

Nejati, M., Rabiei, S., \& Jabbour, C. C. (2017). Envisioning the invisible: Understanding the synergy between green human resource management and green supply chain management in manufacturing firms in Iran in light of the moderating effect of employees' resistance to change. Journal of Cleaner Production, 168, pp.163172.

Ntabe, E. N., LeBel, L., Munson, A. D., \& Santa-Eulalia, L. A. (2015). A systematic literature review of the supply chain operations reference (SCOR) model application with special attention to environmental issues. International Journal of Production Economics, 169, pp.310332.

Ojha, D., Acharya, C., \& Cooper, D. (2018). Transformational leadership and supply chain ambidexterity: Mediating role of supply chain organizational learning and moderating role of uncertainty. International Journal of Production Economics, 197, pp.215-231.

Pai, D. C., Lai, C. S., Chiu, C. J., \& Yang, C. F. (2015). Corporate Social Responsibility and Brand Advocacy in Business-to-Business Market: The Mediated Moderating Effect of Attribution. Journal of Business Ethics, 126(4), pp.685-696.

Partanen, J., Kohtamäki, M., Patel, P.C., \& Parida, V. (2019). Supply chain ambidexterity and manufacturing SME performance: The moderating roles of network capability and strategic information flow. International Journal of Production Economics, https://doi.org/10.1016/j.ijpe.2019.08.005

Peng, J., Quan, J., Zhang, G., \& Dubinsky, J. (2016). Mediation effect of business process and supply chain management capabilities on the impact of IT on firm performance: Evidence from Chinese firms. International Journal of Information Management, 36(1), pp.89-96.

Porter, M.G. (2019). Supply Chain Integration: Does Organizational Culture Matter? Operations and Supply Chain Management, 12(1), pp.49-59.

Pradhan, S. K., \& Routroy, S. (2016). Supply management integration model for Indian manufacturing industries. International Journal of Operations \& Production Management, 36(7), pp.781-802.

Preacher, K.J., Rucker, D.D., \& Hayes, A.F. (2007). Addressing moderated mediation hypotheses: Theory, Methods, and Prescriptions. Multivariate Behavioral Research, 42, pp.185-227.

Ramanathan, R., Ramanathan, U., \& Ko, W. L. (2014). Adoption of RFID technologies in UK logistics: Moderating roles of size, barcode experience and government support. Expert Systems with Applications, 41(1), pp.230236.

Sadiq Jajjaa, M. S., Chathaa, K. A., \& Farooq, S. (2018). Impact of supply chain risk on agility performance: Mediating role of supply chain integration. International Journal of Production Economics, 205, pp.118-138.

Saeeda, A., Malhotrab, K., \& Abdinnour, S. (2018). How supply chain architecture and product architecture impact firm performance: An empirical examination. Journal of Purchasing and Supply Management, 25(1), pp.40-52.

Schniederjans, D. G. (2018). Business process innovation on quality and supply chains. Business Process Management Journal, 24(3), pp.635-651.

Seo, Y. J., Dinwoodie, J., \& Kwak, D. W. (2014). The impact of innovativeness on supply chain performance: is supply chain integration a missing link. Supply Chain Management: An International Journal, 19(5/6), pp.733746.

Sharma, A., \& Nandi, S. (2018). A Review of Behavioral Decision Making in the Newsvendor Problem. Operations and Supply Chain Management, 11(4), pp.200-213.

Shee, H., Miah, S. J., Fairfield, L., \& Pujawan, N. (2018). The impact of cloud-enabled process integration on supply chain performance and firm sustainability: the moderating role of top management. Supply Chain Management: An International Journal, 23(6), pp.500-517.

Shi, Z., \& Liao, Z. (2015). Inter-firm dependence, inter-firm trust, and operational performance: The mediating effect 
of e-business integration. Information \& Management, 52(8), pp.943-950.

Shou, Y., Hu, W., Kang, M., Li, Y., \& Park, Y. W. (2018). Risk management and firm performance: the moderating role of supplier integration. Industrial Management \& Data Systems, 118(7), pp.1327-1344.

Singh, A., \& Teng, T. C. (2016). Enhancing supply chain outcomes through Information Technology and Trust. Computers in Human Behavior. 54, pp.290-300.

Song, M.X., \& Morgan, X. Y. (2019). Leveraging core capabilities and environmental dynamism for food traceability and firm performance in a food supply chain: A moderated mediation model. Journal of Integrative Agriculture, 18(8), pp.1820-1837.

Sreedevi, R., \& Saranga, H. (2017). Uncertainty and supply chain risk: The moderating role of supply chain flexibility in risk mitigation. International Journal of Production Economics, 193, pp.332-342.

Srinivasan, R., \& Swink, M. (2015). Leveraging Supply Chain Integration through Planning Comprehensiveness: An Organizational Information Processing Theory Perspective. Decision Sciences, 46(5), pp.823-861.

Sundram, V. P. K., Bahrin, A. S., Munir, Z. B. A., \& Zolait, A. H. (2018). The effect of supply chain information management and information system infrastructure: The mediating role of supply chain integration towards manufacturing performance in Malaysia. Journal of Enterprise Information Management, 31(5), pp.751-770.

Treiblmaier, H., Mirkovski, K., Lowry, P.B., \& Zacharia, Z.G. (2020). The physical internet as a new supply chain paradigm: a systematic literature review and a comprehensive framework. The International Journal of Logistics Management, 31(2), 239-287.

Uvet, H. (2020). Importance of Logistics Service Quality in Customer Satisfaction: An Empirical Study. Operations and Supply Chain Management, 13(1), pp.1-10.

Vanpoucke, E., Vereecke, A., \& Muylle, S. (2017). Leveraging the impact of supply chain integration through information technology. International Journal of Operations \& Production Management, 37(4), 5 pp.10-530.

Wan, X., \& Sanders, R. (2017). The negative impact of product variety: Forecast bias, inventory levels, and the role of vertical integration. International Journal of Production Economics, 186, pp.123-131.

Wang, L., \& Preacher, K. J. (2015). Moderated Mediation Analysis Using Bayesian Methods. Structural Equation Modeling: A Multidisciplinary Journal, 22(2), pp.249263.

Wang, C., \& Hu, Q. (2017). Knowledge sharing in supply chain networks: Effects of collaborative innovation activities and capability on innovation performance. Technovation, tion.2017.12.002

Wang, M., Jie, F., \& Abareshi, A. (2018). Logistics Capability, Supply Chain Uncertainty and Risk, and Logistics Performance: An Empirical Analysis of Australian Courier Industry. Operations and Supply Chain Management, 11(1), pp.45-54.
Wen, X., Choi, T. M., \& Chung, S. H. (2018). Fashion Retail Supply Chain Management: A Review of Operational Models. International Journal of Production Economics, 207, pp.34-55.

Wichmann, K., Carter, R. \& Kaufmann, L. (2015). How to Become Central in an Informal Social Network: An Investigation of the Antecedents to Network Centrality in an Environmental SCM Initiative. Journal of Business Logistics, 36(1), pp.102-119.

Wiengarten, F., Pagell, M., Ahmed, M. U., \& Gimenez, C. (2014). Do a country's logistical capabilities moderate the external integration performance relationship? Journal of Operations Management, 32(1-2), pp.51-63.

Wieteska, G. (2020). The Impact of Supplier Involvement in Product Development on Supply Chain Risks and Supply Chain Resilience. Operations and Supply Chain Management, 13(4), pp.359-374.

Wong, W.Y., Lai, K. H., \& Bernroider, W. N. (2015). The performance of contingencies of supply chain information integration: The roles of product and market complexity. International Journal of Production Economics, 165, pp.1-11.

Xu, Y., Boh, W. F., Luo, C., \& Zheng, H. (2018). Leveraging industry standards to improve the environmental sustainability of a supply chain. Electronic Commerce Research and Applications, 27, pp.90-105.

Xue, L. (2014). Governance-knowledge fit and strategic risk taking in supply chain digitization. Decision Support Systems, 62, pp.54-65.

Yalcin, H., Shi, W., \& Rahman, Z. (2020). A review and scientometric analysis of supply chain management (SCM). Operations and Supply Chain Management, 13(2), pp.123-133.

Yang, J. (2015). Supply chain agility: Securing performance for Chinese manufacturers. International Journal of Production Economics, 150, pp.104-113.

Yang, D., Sheng, S., Wu, S., \& Zhou, Z. (2018). Suppressing partner opportunism in emerging markets: Contextualizing institutional forces in supply chain management. Journal of Business Research, 90, pp.1-13.

Yoon, S. N., Lee, D., \& Schniederjans, M. (2016). Effects of innovation leadership and supply chain innovation on supply chain efficiency: Focusing on hospital size. Technological Forecasting and Social Change, 113, pp.412421.

Zhang, M., Guo, H., \& Zhao, X. (2017). Effects of social capital on operational performance: impacts of servitisation. International Journal of Production Research, 55(15), pp.4304-4318.

Zhang, Q., \& Cao, M. (2018). Exploring antecedents of supply chain collaboration: Effects of culture and interorganizational system appropriation. International Journal of Production Economics, 195, pp.146-157.

Zhong, J., Ma, Y., Tu, Y., \& Li, X. (2016). Supply chain quality management: an empirical study. International Journal of Contemporary Hospitality Management, 28(11), pp.2446-2472.

Dr. Shu-Hsien Liao is a professor in the Department of Management Sciences, Business and Management College, Tamkang University, Taiwan, Republic of China. He received the Ph.D. degree in Operational Research/System Group of Business 
School, University of Warwick, U.K. His current research interests are in Supply chain management, Data mining and Business intelligence/big data analysis. His publications have appeared in the European Journal of Operational Research, Annals of Operations Research, Journal of the Operational Research Society, International Journal of Production Economics, Decision Support Systems, Information Sciences, Information Processing \& Management, Journal of Information Science, Soft Computing, Applied Intelligence, International Journal of Data Warehousing and Mining, International Journal of Computer Integrated Manufacturing, Journal of Experimental \& Theoretical Artificial Intelligence, Technovation, Knowledge Management Research \& Practice, Total Quality Management \& Business Excellence and Journal of technology transfer.

Dr. Retno Widowati is a Lecture of Department of Management, Universitas Muhammadiyah Yogyakarta, Yogyakarta, Indonesia. Her current research interests include marketing, business/management, supply chain and statistical method. 